\title{
Ampliación del rango altitudinal y primer registro de Dasyprocta punctata (Rodentia: Dasyproctidae), en el Parque Nacional Braulio Carrillo, Sector Volcán Barva, Costa Rica
}

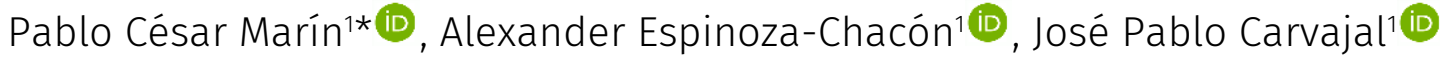 \\ 1 Unlversidad Técnica Nacional, Ingeniería en Manejo Forestal y Vida Silvestre, Sede Atenas, Costa Rica \\ * Correspondencia: pomarin@est.utn.ac.cr
}

\section{Resumen}

Reportamos una ampliación del rango altitudinal de Dasyprocta punctata en Costa Rica. Este resultado, se obtiene a partir de la instalación de cámaras trampa en el Parque Nacional Braulio Carrillo, Sector Volcán Barva, donde se consiguieron diez registros fotográficos de guatusa; sobre los 2.714 msnm, 2.728 msnm y 2.743 msnm. Ampliando en 342 msnm su límite altitudinal y sobrepasando los 2.400 msnm reportados para Costa Rica.

Palabras clave: Dasyprocta punctata, guatusa, Volcán Barva.

Abstract

We report an extension of the elevation range of Dasyprocta punctata in Costa Rica. We obtained this result by placing camera traps in the Braulio Carrillo National Park, Volcano Barva sector. We obtained ten photographic records of guatusa, at elevations of 2.714 masl, 2.728 masl and 2.743 masl. Increasing its altitude limit by 342 masl and exceeding the 2.400 masl previously reported for Costa Rica.

Key words: Dasyprocta punctata, Barva volcano, Central American agouti.

Dasyprocta punctata, conocida comúnmente como guatusa, cherenga o agouti centroamericano, pertenece al orden Rodentia, el más diverso entre los mamíferos del mundo (Wilson \& Reeder 2005; Wallace et al. 2010; Burgin et al. 2018), siendo además el segundo roedor de mayor tamaño en Costa Rica, seguido de Cuniculus paca (Wainwright 2007). Este mamífero mediano tiene hábitos diurnos-crepusculares, donde suele iniciar su actividad desde tempranas horas de la mañana y por lapsos durante el día hasta el anochecer (McLeod 2011). No obstante, la frecuencia y duración de su actividad es dependiente de la abundancia de frutos y semillas de algunas especies como el almendro (Dipteryx panamensis), pejivalle, (Bactris gasipaes), cedro macho, (Carapa guianensis), jobo (Spondias mombin) y guapinol, (Hymenaea courbaril) (Smythe 1978; Wainwright 2007; McLeod 2011; Ramírez et al. 2018). 
La guatusa se distribuye ampliamente por el continente americano, desde el sur de México hasta el sur de Bolivia, norte de Argentina y suroeste de Brasil, alcanzando un rango altitudinal de 3.200 msnm (Morales et al. 2004; Reid 2009; Torres-Trujillo \& Mantilla-Meluck 2017). En los casos de Cuba y en las islas Caimán la presencia de esta especie se le atribuye a que fue introducida (Benavides 2000; Morales et al. 2004). En Costa Rica la guatusa se distribuye en todo el país, con un límite altitudinal de 2.400 msnm (Wainwright 2007).

En este manuscrito presentamos el primer registro de D. punctata, en el Parque Nacional Braulio Carrillo, Sector Volcán Barva; estos reportes se obtuvieron a través de la instalación de doce cámaras trampas de la marca Moultrie y Bushnell, obteniendo 10 registros fotográficos de guatusa, durante los meses de septiembre y octubre del 2016 (Figura 1). Los registros se obtuvieron en tres sectores con diferentes altitudes: sobre los $2.714 \mathrm{msnm}$ (10,1267, -84,1143), 2.728 msnm (10,1267, -84,1187) y sobre los 2.742 msnm $(10,1267,-84,1171)$ (Figura 2). Resulta oportuno destacar, que el Volcán Barva presenta un bosque húmedo siempre verde, con zonas de vida de bosque pluvial montano y bosque pluvial montano bajo (Alvarado 2007). Así mismo, cabe mencionar que las áreas donde se fototrampeó $D$. punctata, se observaron árboles de roble encino (Quercus bumelioides) y sus frutos en el suelo, por lo que es importante indagar si son aprovechadas por esta especie.

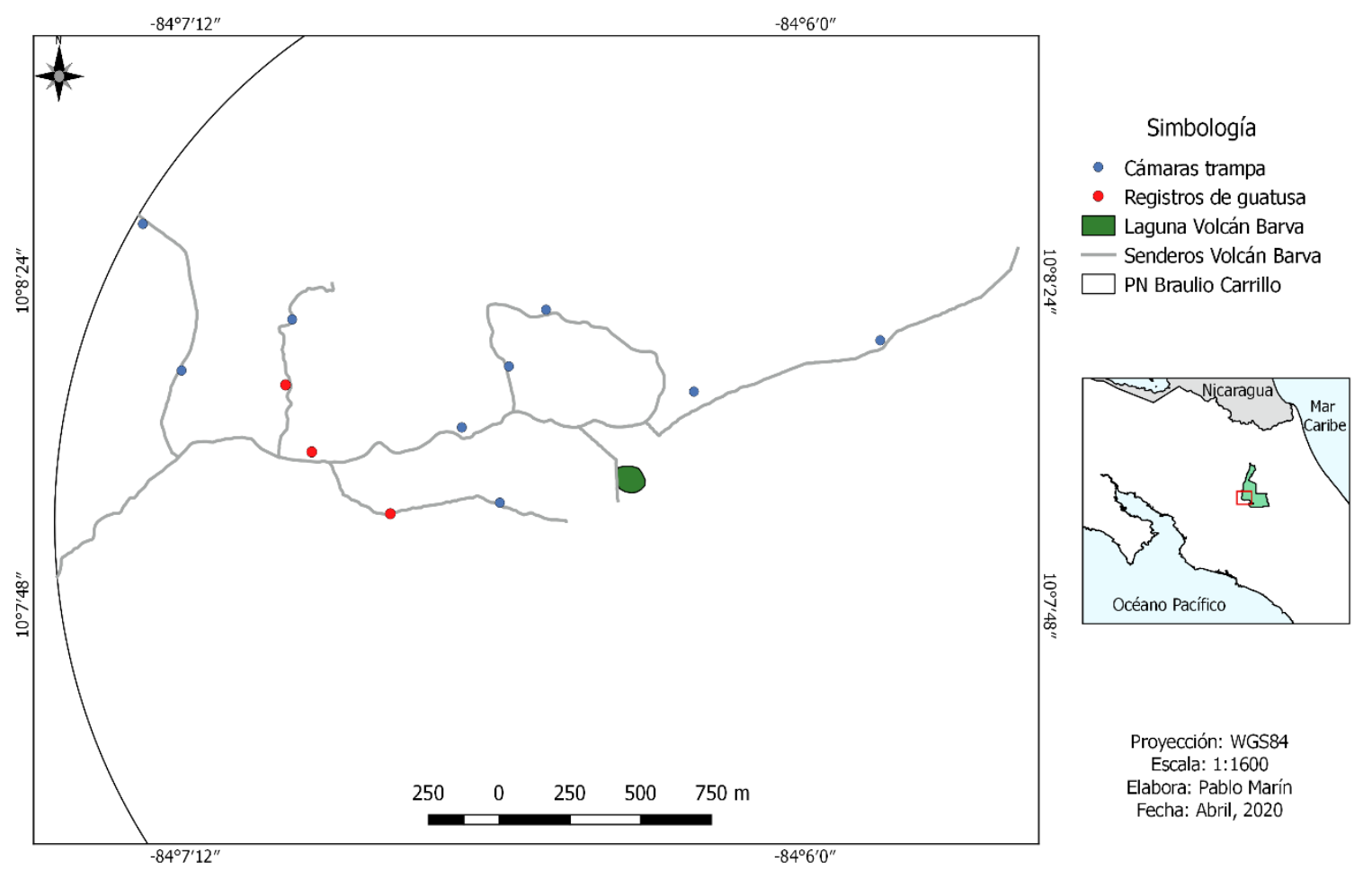

FIGURA 1. Ubicación de los registros de Dasyprocta punctata en el Parque Nacional Braulio Carrillo, Sector Volcán Barva.

El registro de D. punctata en el Volcán Barva, representa un factor importante para los ciclos ecológicos del sitio, ya que contribuyen con la dispersión y depredación de semillas de gran tamaño (Smythe 1978). Asimismo, esta especie es caracterizada por tener la particularidad de enterrar las semillas en periodos de abundancia, con el fin de almacenarlas y consumirlas en épocas de escasez (Wong et al. 1999). Sin embargo, en muchas ocasiones olvidan donde enterraron sus alimentos y las semillas germinan antes de ser aprovechadas, por lo tanto, esta especie contribuye con la regeneración y la 
heterogeneidad espacial del sitio (Wong et al. 1999). Además de lo mencionado, cabe destacar que las guatusas también juegan un rol importante para la dieta de varias especies carnivoras, donde Smythe (1978) reportó que las guatusas son la presa preferida por parte de Leopardus pardalis y Herpailurus yagouaroundi. Adicional a estas especies, también se destacan los reportes de depredación por parte de Panthera onca, Puma concolor, Eira barbara y Nasua narica (Aliaga et al. 2008; Emsens et al. 2013).

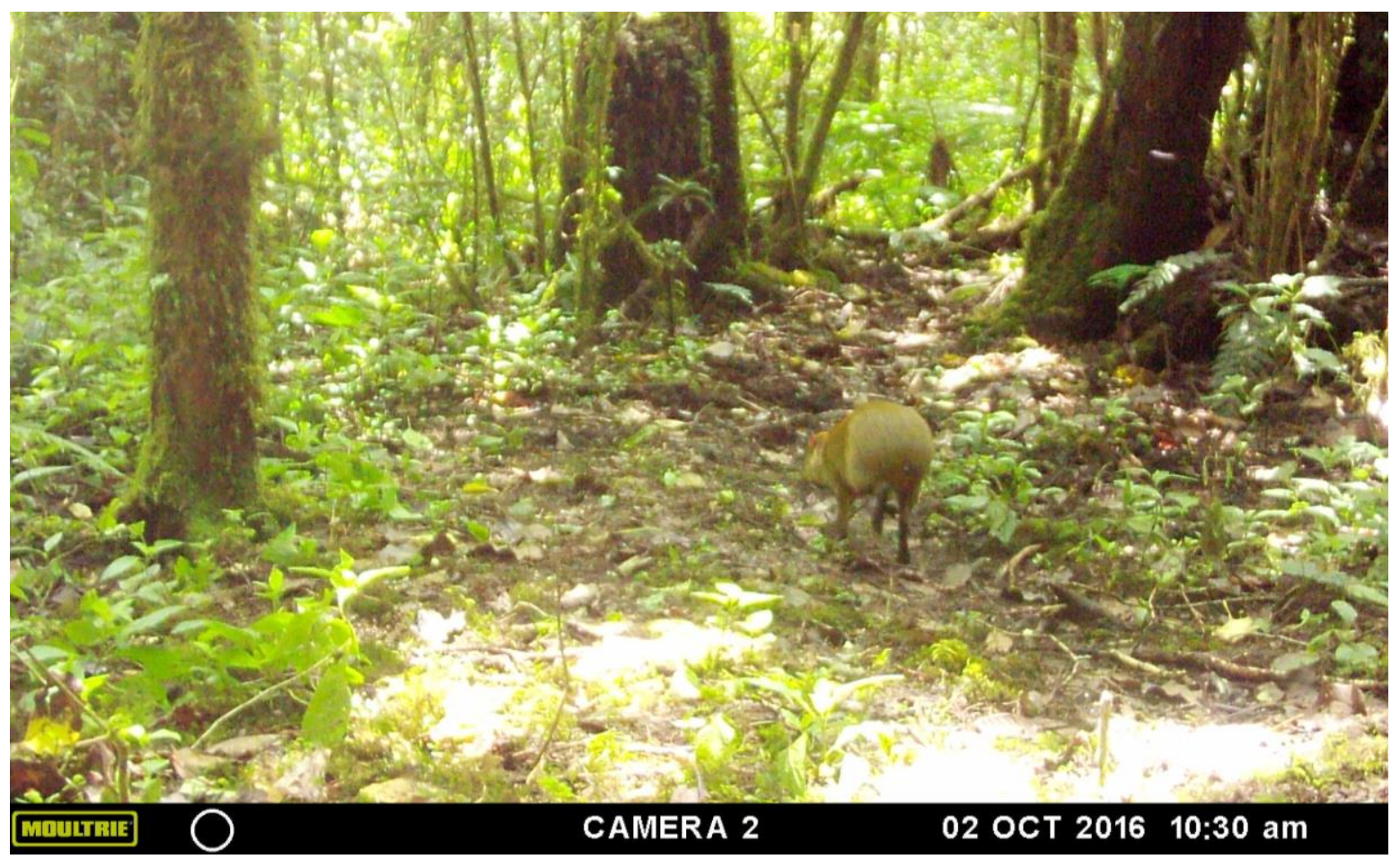

FIGURA 2. Registro de Dasyprocta punctata en el Parque Nacional Braulio Carrillo, Sector Volcán Barva, Costa Rica.

Este nuevo reporte amplía el límite altitudinal de D. punctata en $342 \mathrm{msnm}$, sobrepasando los 2.400 msnm reportados para Costa Rica y América Central (Carrillo et al. 2002; Wainwright 2007; Reid 2009). Por lo tanto, esta evidencia, nos hace pensar que esta especie puede estar ampliando su rango de distribución, en una zona donde no ha sido reportada históricamente (Parque Nacional Braulio Carrillo, Sector Volcán Barva). Por esta razón recomendamos realizar estudios más detallados sobre hábitos alimenticios, patrones de movimiento y selección de hábitat en el sector del Volcán Barva, esto con el fin de ampliar la información sobre la historia natural de la especie.

\section{REFERENCIAS}

Aliaga E, Kays R, Fragoso J. 2008. Home-range use by the Central American agouti (Dasyprocta punctata) on Barro Colorado Island, Panama. Journal of Tropical Ecology. 24(4):367-374. https:// doi.org/10.1017/S0266467408005129

Alvarado J. 2007. Desarrollo ecoturístico en el macizo del Barva. Ambientico, 3-6.

Benavides JM. 2000. Los mamíferos silvestres de Costa Rica. San José, Costa Rica: EUNED.

Burgin CJ, Colella JP, Kahn PL, Upham NS. 2018. How many species of mammals are there?. Journal of Mammalogy 99(1):1-14. https://doi.org/10.1093/jmammal/gyx147. 
Carrillo E, Wong G, Sáenz J. 2002. Mamíferos de Costa Rica. Santo Domingo, Heredia, Costa Rica: Instituto Nacional de Biodiversidad (INBio).

Emsens W, Hirsch B, Kays R, Jansen P. 2013. Prey refuges as predator hotspots: ocelot (Leopardus pardalis) attraction to agouti (Dasyprocta punctata) dens. Acta Theriologica, 59(2):4-8. https:// doi.org/10.1007/s13364-013-0159-4

Mcleod EF. 2011. Dispersión de semillas por mamíferos terrestres en bosques latifoliados del Atlántico Norte de Nicaragua después del huracán Félix. Recursos Naturales y Ambiente, 62: 37-46.

Morales A, Sánchez F, Poveda K, Cadena A. 2004. Mamíferos terrestres y voladores de Colombia. Bogotá, Colombia:Ramos López editorial.

Ramírez Calvo D, Rivera Vargas A, Villalobos Molina H, Marin Solano M, Valverde Cordero L, Acosta Chaves VJ .2018. Depredación oportunista sobre Turdus grayi (Passeriformes: Turdidae) por Dasyprocta punctata (Rodentia: Dasyproctidae) en San Vito, Costa Rica. Mammalogy Notes. 4(2):11-12.

Reid F. 2009. A field guide to the mammals of Central America and Southeast Mexico. $2 \mathrm{~d}$ ed. Oxford University Press, New York, US.

Smythe N. 1978. The Natural History of the Central American Agouti (Dasyprocta punctata). Smithsonian Contributions to Zoology 257:1-52.

Torres-Trujillo N, Mantilla-Meluk H. 2017. Común e ignorado: ausencia de documentación científica del guatín Dasyprocta punctata (Rodentia: Dasyproctidae) en el departamento del Quindío, Colombia. Biodiversidad Neotropical. 7(1):30-8. http://dx.doi.org/10.18636/bioneotropical.v7i1.588

Wainwright M. 2007. The mamals of Costa Rica a natural history and field guide. Zona Tropical Publications, 1st edition, New York, USA.

Wallace R, Gómez H, Porcel Z, Rumiz D. 2010. Distribución, Ecología y Conservación de los mamíferos medianos y grandes de Bolivia. Bolivia: Centro de Ecología Difusión Simón Patiño.

Wilson DE, Reeder DM. 2005. Mammal species of the world: a taxonomic and geographic reference (Vol. 2). JHU Press.

Wong G, Sáenz J, Carrillo E. 1999. Mammals of Corcovado National Park. Santo Domingo, Heredia, Costa Rica: Instituto Nacional de Biodiversidad (INBio).

Editor: Diego J. Lizcano

Recibido: 2020-04-08

Revisado: 2020-04-12

Aceptado: 2020-05-15

Publicado: 2020-06-22 